\title{
Hydraulics and heat transfer in the IFMIF liquid lithium target: CFD calculations
}

\author{
A. Peña ，G.A. Esteban , J. Sancho , V. Kolesnik , A. Abánades \\ University of the Basgue Country, Nuclear Engineering and Fiuid Mechanics Department, Alameda de Urquijo s/n, 48013 Bilbao, Spain \\ ZAO "EMT-R" 46, Suzdaiskaya Street, 111672 Moscow, Russia \\ Polytechnic University of Madrid, Power Engineering and Fiud Mechanics Department, José Gutierrez Abascoi 2, 28006 Madrid, Spain
}

\begin{abstract}
A B S T R A C T
CFD (Computational fluid dynamics) calculation turns out to be a good approximation to the real behavior of the lithium (Li) flow of the target of the international fusion materials irradiation facility (IFMIF). A three-dimensional (3D) modelling of the IFMIF design li target assembly, made with the CFD commercial code ANSYS-FLUENT has been carried out. The simulation by a structural mesh is focused on the thermalhydraulic analysis inside the Li jet flow. For, this purpose, the two deuteron beams energy deposition profile is modelled as an energy source term inside the volume of liquid affected. Turbulence is estimated using the RNG $k-\varsigma$ model, and a surface-tracking technique applied to a fixed Eulerian mesh called volume of fluid (VOF) is used to determine the position of the free surface. Calculations varying the jet velocity from a range of $10-20 \mathrm{~m} / \mathrm{s}$, show that maximum calculated temperatures are still below the lithium's boiling point, due to the increase of the pressure induced by centrifugal force.
\end{abstract}

\section{Introduction}

The international fusion materials irradiation facility (IFMIF) is currently being planned by Japan, Russia, the EU, and the United States [1]. and it is an accelerator-based deuteron-lithium neutron source thought to produce intense high energy neutrons for testing fusion materials to be used in ITER and fusion DEMO reactor. Two deuteron beams at an energy of $40 \mathrm{MeV}(2 \times 125 \mathrm{~mA})$ will be injected into a high-speed liquid lithium ( $\mathrm{Li}$ ) jet, flowing along a vertical concave wall in a $10^{-3}$ Pa vacuum. An average surface heat flux of $1 \mathrm{GW} / \mathrm{m}^{2}$ on the Li free surface will be produced by the irradiation of the beam.

The IFMIF Li target assembly is designed to provide a stable $\mathrm{Li}$ jet in a safe operation mode. Great waves could have effects on the neutron field, and there could be a possibility of wall burning by the beams. On the other hand, high velocities up to $20 \mathrm{~m} / \mathrm{s}$ are needed to remove the deposited beam energy. Table 1 shows the IFMIF main specifications to be taken into account for the numerical simulation.

In Fig. 1, a scheme of the IFMIF design allows a general description of the main features involved in the target flow: the concave back-wall, the li free surface, the vacuum zone and the beam impact zone.

The concave back wall, whose radius is $25 \mathrm{~cm}$ avoids bi boiling because there is a centrifugal induced overpressure [2].
The simulation of working conditions with the available engineering computational fluid dynamic (CFD) codes is part of the design work, and the validation of such codes will always be the proper way to improve them and to rely on their predicted results.

This study shows calculations made with the CFD commercial code ANSYS-FLUENT [3], focused on the thermal-hydraulic analysis of technology issues such as a vacuum environment, a high heat energy source, a liquid metal flow, and a free surface flow. Considerations are made about the capabilities and deficiencies of the models included in ANSYS-FLUENT when dealing with the mentioned physical phenomena.

\section{Thermal-hydraulic analysis with ANSYS-FLUENT CFD code}

\subsection{Grid and physical models}

The geometry grid for the IFMIF target simulation is shown in Fig. 2. A meshing of 881,160 hexahedral cells has been used for the simulation. The grid density of the region between the concave wall and up to $25 \mathrm{~mm}$ from it, is 20 elements in perpendicular direction from the wall. Therefore, cells are $1.25 \mathrm{~mm}$ high in that direction. The first cell next to this zone is $3.8 \mathrm{~mm}$ high. This cell structure is maintained down to the outlet.

A two phase flow problem has to be solved: Lithium and a vacuum environment. This last one being air at $10^{-3} \mathrm{~Pa}$. One of the main concerns about this flow is the stability of the liquid metal free surface. To track this surface, CFD codes as ANSYS-FLUENT use a surface-tracking technique applied to a fixed Eulerian mesh 
Table 1

IFMIF target specification.

\begin{tabular}{ll}
\hline Area of the Li Jet (width $\times$ thickness) & $260 \mathrm{~mm} \times 25 \mathrm{~mm}$ \\
Velocities of the Li jet & A range from $10-20 \mathrm{~m} / \mathrm{s}$ \\
Inlet temperature of Li & $250^{\circ} \mathrm{C}$ \\
Pressure at free surface (vacuum) & $10^{-3} \mathrm{~Pa}$ \\
Energy (beam) deposition surface & 200 (width) $\times 50 \mathrm{~mm}$ (height) \\
\hline
\end{tabular}

called volume of fluid (VOF). It is designed for two or more immiscible fluids where the position of the interface between the fluids is of interest. This is the case, as a Lithium jet flows in a vacuum environment along a concave wall.

The volume fraction for phase $q\left(\alpha_{q}\right)$ is calculated through the transport Eq. (1) [3]:

$\frac{\partial\left(\alpha_{q} \rho_{q}\right)}{\partial t}+\nabla\left(\alpha_{q} \rho_{q} \vec{v}\right)=S_{\alpha_{q}}+\sum_{p=1}^{n}\left(\dot{m}_{p q}-\dot{m}_{q p}\right)$

where $S_{\alpha_{q}}$ is the source term for the volume fraction for phase $q$. By default this source term is zero. $\dot{m}_{q p}$ is the mass transfer from phase $q$ to phase $p$, and $\dot{m}_{p q}$ is the mass transfer from phase $p$ to phase $q$.
The volume fraction for the other phases must follow the expression (2):

$$
\sum_{q=1}^{\pi} \alpha_{q}=1
$$

The material properties, $\varphi$, in one computational cell are calculated as average values of each phase properties, using Eq. (3):

$\phi=\sum \alpha_{q} \phi_{q}$

Therefore, if there is only one phase in that cell, the property will correspond to that phase. Discretization of the transport equation has been done with an explicit time scheme. For this scheme the default Courant number is 0.25 , and it is the value used for the calculations. The time step for VOF calculation is refined, based on this maximum Courant number allowed near the free surface. It compares the time step in the calculation with the characteristic time of transit of a fluid element across a control volume. This time is calculated, in the region near the fluid interface, dividing the volume of each cell by the sum of the outgoing fluxes. The convergence criterion for the transport equation is also the default one, $10^{-3}$.

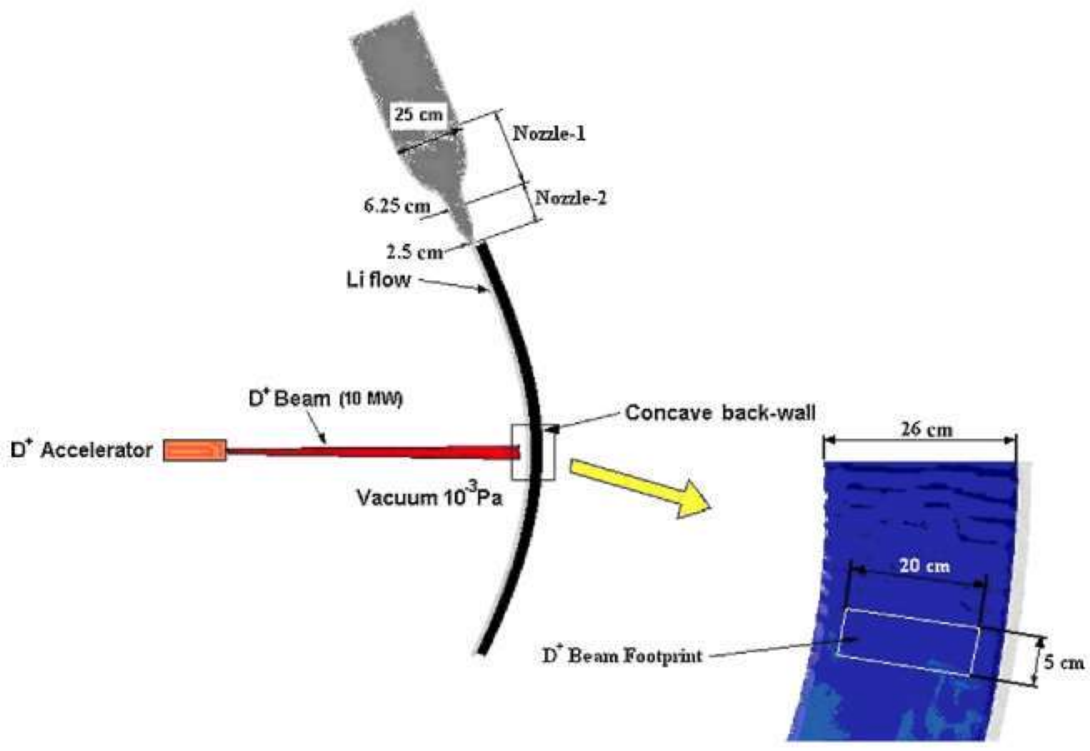

Fig. 1. Scheme of the IFMIF target geometry.

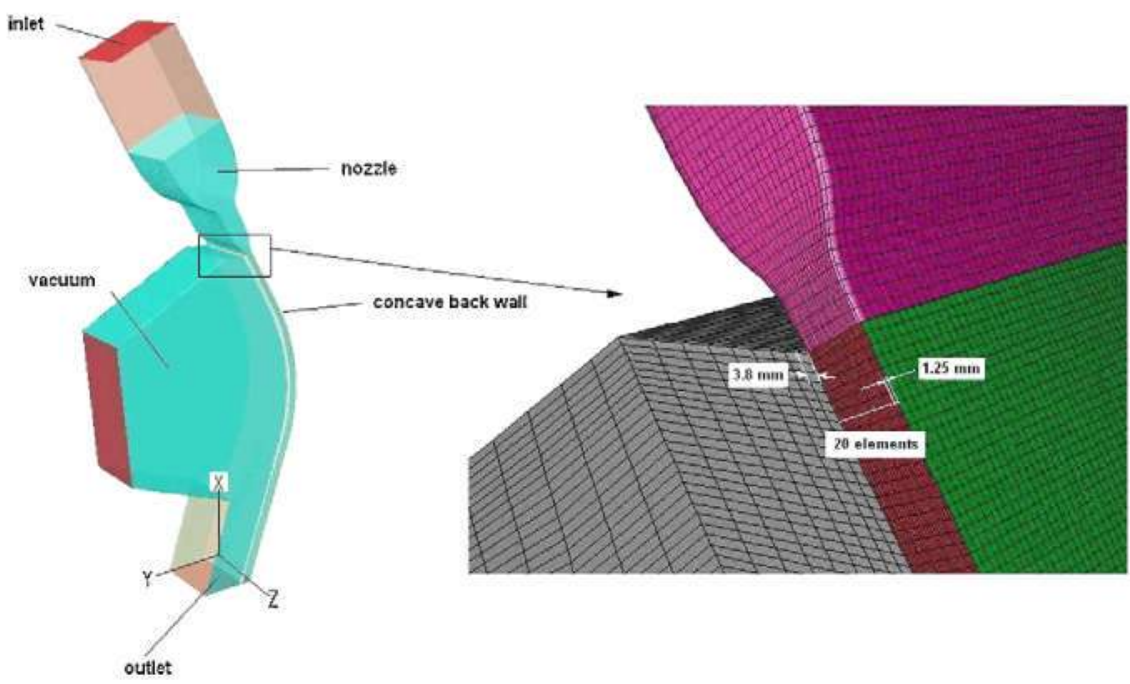

Fig. 2. IFMIF target geometry. 
Then, the interface shape is calculated through the geometric reconstruction scheme. First of all the position of the linear interface is located relative to the center of each partially filled cell. This is done based on information about the volume fraction and its derivatives in the cell. Then, for the fluxes of fluid through each face the computed linear interface and the information about the normal and tangential velocity distribution on the face are used. With these fluxes, the volume fraction in each cell is calculated.

One momentum equation is applied in the whole domain, and it depends on the phase volume fractions through the density and the molecular viscosity:

$\frac{\partial}{\partial t}(\rho \overrightarrow{\mathbf{v}})+\nabla(\rho \overrightarrow{\mathrm{v}})=-\nabla p+\nabla\left[\mu\left(\nabla \bar{v}+\nabla \bar{v}^{T}\right)\right]+\rho \overrightarrow{\mathrm{g}}+\vec{F}$

The surface tension is taken into account as a source term in the momentum equation, $F$, and is evaluated using $\mathrm{Eq}$. (5), based on the continuum surface force (CSF) formulation [4]:

$F=\sum_{\text {pairspg }} \sigma_{p q} \frac{\alpha_{p} \rho_{p i} \kappa_{q} \nabla \alpha_{q}+\alpha_{q} \rho_{q} \kappa_{p} \nabla \alpha_{p}}{\frac{1}{2}\left(\rho_{p}+\rho_{q}\right)}$

where $\sigma_{p q}$, is the surface tension between phases $q$ and $p . \kappa$, is the curvature, which is the divergence of the unit surface normal. If only two phases are present, as in this case, $\kappa_{t}=-\kappa_{p}$.

Turbulence is also a main key for this kind of patterns. The model chosen for the calculations is the RNG $k-\varepsilon$ model, as its good physical properties and less computational cost allow a suitable simulation time. Other models, with the number of cells mentioned before, and a multiphase flow involved, are, by the moment, of a very expensive computational cost. However, in these calculations, the effective viscosity ( $\mu_{\text {elf }}$ ) variable to be introduced into the $k$ and $\varepsilon$ transport equations is obtained through a differential Eq. (6),

$d\left(\frac{\rho^{2} k}{\sqrt{\varepsilon \mu}}\right)=1.72 \frac{\hat{v}}{\sqrt{\hat{v}^{3}-1+C}} d \hat{\imath}$

and not by the standard set. Therefore, the turbulence will vary with the eddy scale, being $\hat{v}=\mu_{\text {eft }} / \mu$ and $C_{v}=100$ [3].

Heat transfer has been modelled using the Reynolds' analogy, but for the RNG model, the effective thermal conductivity used in the energy equation is:

$k_{\mathrm{eft}}=\alpha c_{p} / \mu_{\mathrm{etf}}$

where $\alpha$ (inverse of the turbulent Prandtl number) is calculated from Eq. (8):

$\left|\frac{\alpha-1.3929}{\alpha_{0}-1.3929}\right|^{0.6321}\left|\frac{\alpha+2.3929}{\alpha_{0}+2.3929}\right|^{0.3679}=\frac{\mu}{\mu_{\mathrm{eff}}}$

with $\alpha_{0}=1 / \mathrm{Pr}=k / \mu c_{p}$

Therefore, the turbulent Prandtl number is not constant as in the standard $k-\varepsilon$ model, but it depends on the molecular Prandt] number.

The standard wall functions have been used, as grid requirements for enhance wall treatment by ANSYS-FLUENT (using the correlation proposed by Kader [3]) could not be managed by available computational resources. In these wall functions turbulent Prandtl number is constant with a value of 0.85 .

\subsection{Boundary conditions and discretization schemes}

Wall conditions have been used for the sides of the domain, considering the test section as a whole. Then, a uniform velocity profile is the input for the inlet, and an absolute pressure of $10^{-3} \mathrm{~Pa}$ at the outlet (Fig. 1)

The energy deposition by the $\mathrm{D}^{+}$beams is simulated by an ene rgy source $\left(\mathrm{W} / \mathrm{m}^{3}\right)$ input within a volume in the Lithium phase in the

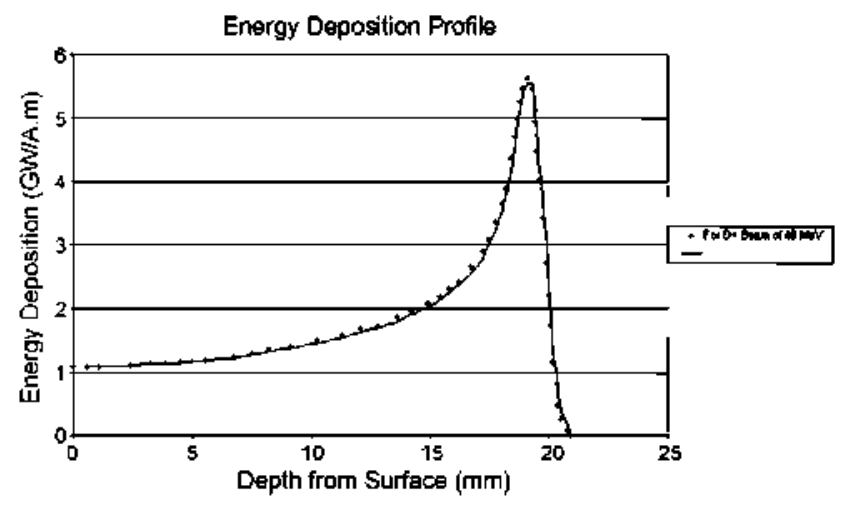

Fig. 3. Energy deposition profile.

middle part of the domain, (see Fig. 1). ln Fig. 3 can be seen the energy profile used for the calculations (taken from [2]).

A second order UPWIND discretization scheme has been used for momentum, turbulent kinetic energy, turbulent dissipation rate, and energy.

\subsection{Calculation analysis}

The thermal-hydraulic characteristics of the liquid flow has been evaluated for two velocities: $10 \mathrm{~m} / \mathrm{s}$ and $20 \mathrm{~m} / \mathrm{s}$. Unsteady formulation needed for VOF calculations was used. Then, the problem stopped when a steady solution was reached.

ANSYS-FLUENT predicts that $\mathrm{Li}$ boiling point is not reached inside the domain. Fig. 4, shows that, the maximum temperature reached inside the lithium phase is $415^{\circ} \mathrm{C}$, for a jet velocity of $20 \mathrm{~m} / \mathrm{s}$, and in the case of $10 \mathrm{~m} / \mathrm{s}$, calculations show a maximum temperature of $586^{\circ} \mathrm{C}$, and boiling point is around $990^{\circ} \mathrm{C}$. The centrifugal force effect of increasing the boiling point of the fluid has turned out to be effective, as the boiling point in that part of the domain is around $1090^{\circ} \mathrm{C}$ [5], using the formula (9):

boiling point $=\frac{17900}{22.7-\log P(P a)}-262$

At the free surface, being the pressure $10^{-3} \mathrm{~Pa}$, the boiling point is $344^{\circ} \mathrm{C}[6]$. Therefore the temperature margin is smaller at the free surface ( $94^{\circ} \mathrm{C}$, predicted by ANSYS-FLUENT) than inside the $\mathrm{Li}$ flow.

Also in Fig. 4, can be seen that the two cells next to the wall are still with a temperature of $250^{\circ} \mathrm{C}$ (the inlet temperature). That means higher temperatures are $2.5 \mathrm{~mm}$ from the wall, being the maximum temperature $6 \mathrm{~mm}$ from the wall.

It is a well known deficiency of most turbulence models used in commercial CFD codes (included the RNG $k-\varepsilon$ ), when dealing with liquid metals, that they assume the Reynolds analogy, using the turbulent Prandtl number to describe turbulent heat transport [6]. That seems not to be valid for low-Prandtl number liquids (high thermal conductivity, like liquid metals). In particular, near wall treatment should be reviewed. These models consider direct proportionality between turbulent moment transport and turbulent heat transport. As mentioned before, the turbulent Prandtl number used in the temperature wall functions is by default 0.85 . That means that turbulent moment and turbulent heat transport are equivalent. This result is suitable for other liquids like water or air. but not for liquid metals. Using this value leads to an overestimation of turbulent heat flux. which in the case of liquid metals seem to be negligible.

The velocity field and the grid resolution considered in these calculations lead to $y+$ values up to 300 , so, at the nozzle no relaminarization of the boundary layer has been observed. 


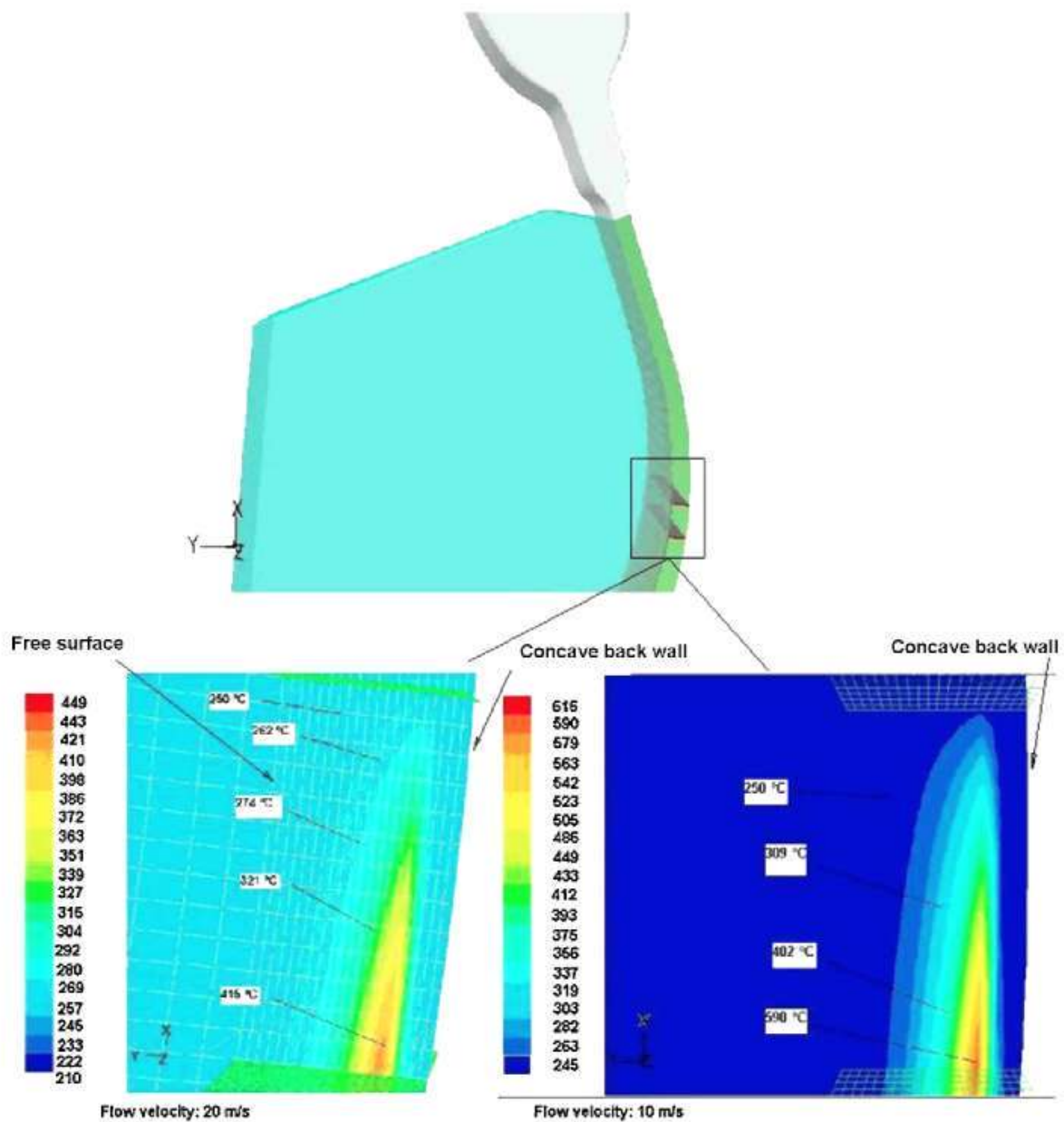

Fig. 4. Temperature distribution in the energy-deposition zone, for a jet velocity of $20 \mathrm{~m} / \mathrm{s}$.

Surface fluctuations observed in the calculations are caused by waves, thought to be due to turbulence and are transverse to the flow. In fact their peak-to-peak amplitude is slightly higher when velocity increases. Fig. 5 shows the calculated surface waves formed when the Li flow velocity is $20 \mathrm{~m} / \mathrm{s}$. The maximum peak-to-peak amplitude of these waves predicted by ANSYS-FLUENT is around $2.5 \mathrm{~mm}$ when the velocity is $20 \mathrm{~m} / \mathrm{s}$, and a little bit smaller, $2 \mathrm{~mm}$, when velocity is $10 \mathrm{~m} / \mathrm{s}$.The wakes are due to impurities (chemical compounds) attached at the nozzle edges and are longitudinal to the flow. They might also be due to imperfections on the nozzle surface (effect of corrosion or erosion).

Fig. 5 also shows that in the most vertical zone of the concave wall (where the energy-deposition zone is located), waves are smaller.

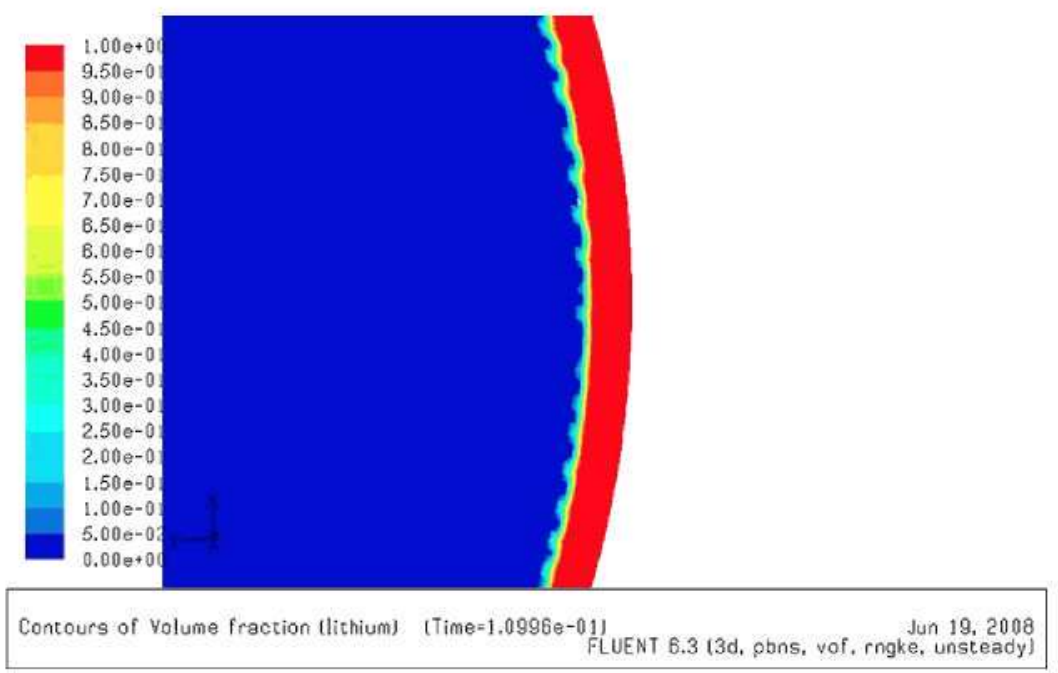

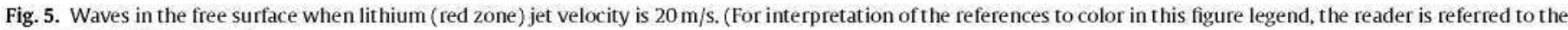
web version of the article.) 


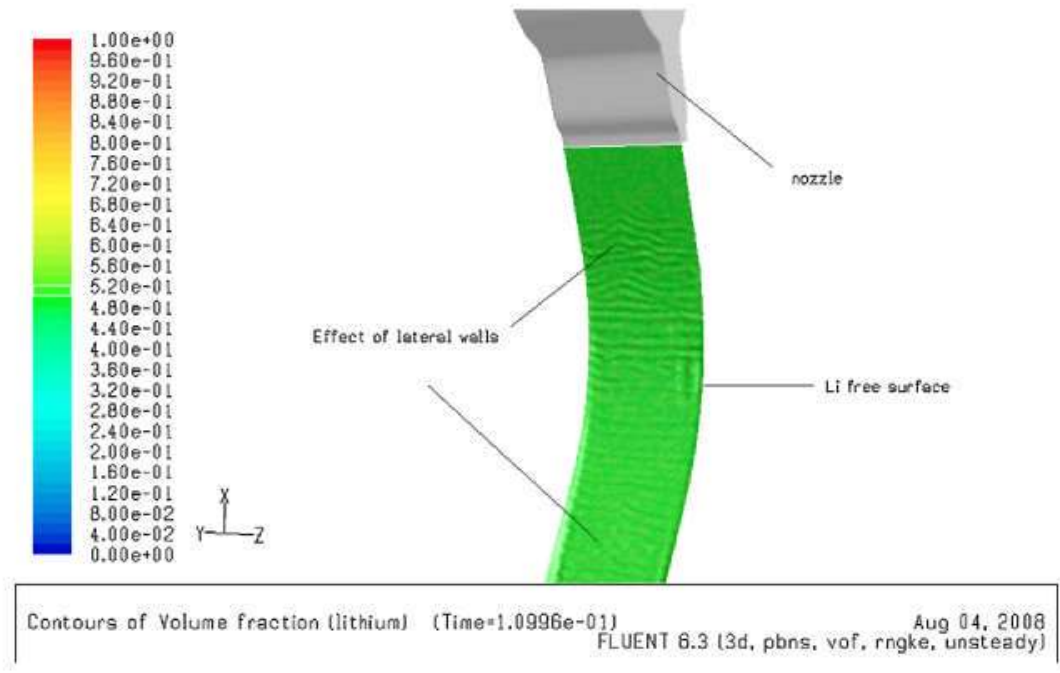

Fig. 6. Effect of lateral walls on the transversal waves, for an average velocity of $20 \mathrm{~m} / \mathrm{s}$.

The effect of lateral walls would lead to the presence of swirlwaves interfering with transversal waves. This could be depicted in Fig. 6.

At this state of the calculations other turbulence phenomena as Götler vortices that appear at curve walls were not studied. Besides, the steady state reached by the calculations and the modellation of the turbulence do not allow to test the formation and cancellation of these vortices and their contribution to Reynolds stress. Direct numerical simulation could be the way to identify these vortices, if computational resources allow the performance.

On the other hand, previous calculations made with a coarser grid could not predict the presence of any kind of waves. This coarser grid density was for the region between the concave wall and up to $25 \mathrm{~mm}$ from it, 10 elements in perpendicular direction from the wall. Therefore, cells were $2.50 \mathrm{~mm}$ high in that direction, and the first cell next to this zone was $7.6 \mathrm{~mm}$ high. This cell structure is maintained down to the outlet.

\section{Conclusion}

Thermal-hydraulic analysis predicts a Li flow without boiling, with a margin of $94^{\circ} \mathrm{C}$, when the concave wall radius is $25 \mathrm{~cm}$. When modelling thermal effects, turbulence plays a very important role. In the case of liquid metal flows, turbulent heat flux seems to be negligible due to the high thermal conductivity of liquid metals. But commercial codes consider that this effect is important, leading to an overestimation of turbulent heat flux. Therefore, other turbulent models should be used, or a suitable correlation should be found for the experimental coefficient involved in this phenomenon, the turbulent Prandtl number.
Also wave formation is due to turbulence phenomena, therefore, the validation of a suitable turbulent model with liquid metals flows should be a priority for CFD code users and developers involved in nuclear devices design.

From the ASCHLIM project it could be observed that the main shortcoming of CFD codes when dealing with liquid metals were the turbulence and the multiphase models. In this paper this two models play a main role in the calculations. However, there is still much work to be done in these fields.

\section{Acknowledgements}

This work has been funded by the University of the Basque Country.

The authors would like to thanks the useful remarks made by Alex Mikheev.

\section{References}

[1] IFMIF Comprehensive Design Report, IFMIF International Team, Published from Foschungszentrum Karlsruhe-IRS, January 2004.

[2] M. Ida, H. Nakamura, H. Nakamura, H. Nakamura, K. Ezato, H. Takeuchi, Thermalhydraulic characteristics of IFMIF liquid lithium target, Fusion Engineering and Design 63-64 (2002) 333-342.

[3] ANSYS-FLUENT 6.3, Theory Manual, Fluent Inc. Central Source Park 10, Cavendish Court, Lebanon, NH, USA, 2005, http://www.fluent.com.

[4] J.U. Brackbill, D.B. Kothe, C. Zemach, A continuum method for modeling surface tension, Journal of Computational Physics 100 (1992) 335-354.

[5] H. Nakamura, M. Ida, H. Nakamura, H. Takeuchi, IFMIF international team. Liquid lithium target under steady state ultra high heat load of $1 \mathrm{GW} / \mathrm{m}^{2}$ range for the international fusion materials irradiation facility (IFMIF), Fusion Engineering and Design 65 (2003) 467-474.

[6] ASCHLIM (Assessment of Computational Fluid Dynamics codes for Heavy Liquid Metals) Project No, FISS-2001-0001. European Community. Euratom FP5 Programme (1998-2002), Final Technical Report, 2003. 